\title{
Trends in Pharmacogenetics
}

\author{
Shweta S Joshi and Husayn Ahmed P* \\ National Centre for Biological Sciences, India
}

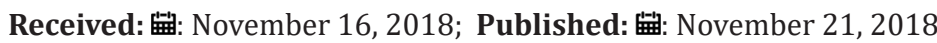

*Corresponding author: Husayn Ahmed P, National Centre for Biological Sciences, India

\begin{abstract}
Since the initial studies in the 1950s, the field of pharmacogenetics has shown a lot of promise. The advent of high-throughput technology and next-generation sequencing has only added to the development of this field. Amidst several challenges like the lack of a comprehensive genomic database and varied prescription practices, pharmacogenetics has immense potential from the personalized medicine, novel biomarker discovery and drug development perspective in a genetically diverse population like India.
\end{abstract}

Keywords: EMEA: European Agency For Evaluation of Medicinal Products, ADRS: Adverse Drug Reactions, PD: Pharmacodynamics, PK: Pharmacokinetics, CPIC: Clinical Pharmacogenetics Implementation Consortium

\section{Introduction}

The concept of tailor-made therapy for everyone has gained a lot of momentum in the last few years. This emerging concept termed "personalized medicine" revolves around the idea of providing individual-specific tailored medical treatment based on the characteristics of the patient, that includes their genetic makeup, lifestyle and other factors. The central core of personalized medicine involves predicting the response of various drugs or therapies on different groups of people [1]. These groups can be defined based on a large spectrum of factors, from their geographic locations and ethnicity to their genotypes and phenotypes. A new scientific discipline termed "pharmacogenetics" came into existence to enable the personalized medicine. This review aims to provide a brief history of pharmacogenetics and a related field pharmacogenomics. This review also tries to highlight the trends of pharmacogenetics in India, the potential applications of this field and the challenges faced in the application at the clinics.

\section{Pharmacogenetics in a Nutshell and its History}

"Pharmacogenetics" has been widely defined as the study of variable drug response based on genetic polymorphisms. Another term that is used interchangeably with pharmacogenetics in "pharmacogenomics". In 2002, The European Agency for Evaluation of Medicinal Products (EMEA) defined "pharmacogenetics" as "the study of inter-individual variations in DNA sequence related to drug response" and "pharmacogenomics" as "the study of the variability of the expression of individual genes relevant to disease susceptibility as well as drug response at cellular, tissue, individual or population level". Evidence of pharmacogenetics can be traced back to 510 B.C. Pythagoras was the first to observe the extent of variability of adverse drug reactions (ADRs) in individuals on consumption of fava beans. Substantial evidence emerged in the 1950s, that associated ADRs to the deficiency of an enzymatic activity. However, the term "pharmacogenetics" was coined by a German geneticist, Friedrich Vogel in 1959. Advances in genetic technologies through late 20th and early 21st century have been instrumental in identifying many genetic variations that may lead to an inter-individual variability in drug response. The completion of the Human Genome Project [2], the HapMap Project [3] and the 1000 genome project [4] have contributed substantially to the rapid expansion of this field.

The genetic variants influencing absorption, distribution, metabolism and elimination of drugs are the most important pharmacogenetic biomarkers. A drug response is based on the pharmacokinetics (PK) (adsorption, distribution, metabolism and elimination), pharmacodynamics (PD) (effect of the drug or metabolites on their target in the body) or in some cases, a combination of the two [5]. Variation in genes involved in these processes may lead to exposure dependent (Type A) ADRs. However, ADRs may also be idiosyncratic [6]. While genetic factors need to be used in tandem with non-genetic factors (e. g. age, body composition, lifestyle, family history) to predict drug response, genetic variations alone, in VKORC1 and CYP2C9 account for 35$50 \%$ variability in warfarin dosage [7]. Based on an overwhelming amount of evidence, Clinical Pharmacogenetics Implementation Consortium (CPIC) of the Pharmacogenomics Research Network was established to provide systematic guidelines for the 
prescription of actionable drugs, pharmacogenetic tests and their translation in the clinic. Currently, CPIC lists guidelines of 35 druggene pairs [8]. Pharm GKB is another publicly available database of genetic variation and drug response.

\section{Need for Pharmacogenetics in the Indian Context}

India is home to $17.5 \%$ of the world population and is one of the top five global pharmaceutical markets as of 2018. One of the biggest challenges and financial burden that the pharmaceutical industry is facing is the high drug attrition rate. In a study concluded in 2015 found that of the 640 novel drugs that entered phase three trials between 1998 and 2008, 54\% failed [9]. A recent survey of drug discovery and development trends in India indicated that of the 214 drugs that entered preclinical development between 1994 and mid-2016, only one was eventually launched [10]. High toxicity because of unpredictable bioavailability of the molecule and lack of efficacy some of the most cited reasons for drug failure during development stages [11]. Even after extensive clinical trials, drugs are often withdrawn from the markets because of reports of ADRs in some populations. Serious incidences of ADRs are observed in $6.7 \%$ of hospital admissions in India [12]. The challenges faced during drug development and in the form of adverse drug reactions and inconsistencies in response can be correlated with the diverse allele frequency distribution of drug metabolizing enzymes (CYP450s, TPMT, GSTs, COMT, SULT1A1, NAT2 and UGTs) and drug transporters (MMDR1, OCT1 and SLCO1B1) amongst the various Indian sub-populations (North Indians, South Indians and North East Indians) [13].

\section{Potential Applications and Challenges}

The most important contribution of this emerging field is the prediction of drug response based on genotype information. This had not only helped accurate estimation of dosage but also supplements the accelerated discovery of novel biomarkers. Earlier this used to be strenuous trial and error procedure, which involved a very high risk of severe adverse reactions in some patients. In some cases, genetic information about drug response has been used to repurpose a drug in specific patient groups. This drug response-genotype information can be leveraged by drug development groups to stratify subjects for clinical trials. When treatments are being personalized for a certain population or certain group of individuals, the inputs from the pharmacogenetic studies prove to be very valuable. There are commercially available tests that can detect a specific set of genetic variations and predict how the patient is likely to respond to certain drugs. While there are many hurdles when translating personalized medicine in the clinics, there also are several challenges conducting, producing and replicating reliable results from pharmacogenetic studies.

First, the accurate genotyping of individuals is essential. The techniques employed to identify genetic variations in individuals are not without errors. These studies rely on either hybridizationbased microarray chip or sequencing-based techniques to identify genotypes. While hybridization-based techniques are prone to higher levels of false discovery rates, there are errors, though in smaller numbers, in sequencing-based techniques too [14]. Therefore, it's necessary to confirm the level of confidence with which the genotypes are identified which are correlated to the variability in the drug response. Those variations need to be confirmed experimentally or in a different platform. The current sequencing strategies also, do not take into consideration rare variants in drug metabolizing genes because of several technical limitations [15].

Second, the pharmacogenomic studies not only stop at identifying the SNPs and correlating them to the drug response, but they also try to identify the haplotypes (SNPs that are co-located and/or statistically associated) [16]. The HapMap project has provided a very significant resource which can be used to identify haplotypes in the individuals but its usefulness in all populations is still debatable. For a population like India, with a very high genomic distinctiveness, these resources fail to provide the correct reference sets that can be used to identify correct haplotypes. While the south Asian subset of HapMap helps for such studies in India, the confidence with which the haplotypes are identified and correlated are not accurate [17]. Third, several large-scale pharmacogenomic studies in each population are required to create a comprehensive and reliable resource that could form the backbone of personalized medicine for that population. Internationally, some of the major hindrances in the widespread adoption of pharmacogenetic tests have been a knowledge gap amongst the physicians and the lack of a decision support mechanism [18].

\section{Conclusion}

Although the field of pharmacogenetics is in its nascent stages in India, there is tremendous potential for exploration. The geographical location and ancestry based diverse genetic polymorphisms observed in the drug metabolizing genes and other proteins influencing the pharmacodynamic and pharmacokinetics of the drug make the widespread adoption of pharmacogeneticsbased techniques extremely challenging. There is a need for a detailed and region-specific genetic database, convenient and affordable genetic testing as well as the development of an interface that works towards informing physicians about pharmacogeneticsbased prescription practices.

\section{References}

1. Vogenberg FR, Isaacson Barash C, Pursel M (2010) Personalized medicine: part 1: evolution and development into theranostics. P \& T: a peer-reviewed journal for formulary management 35(10): 560-576.

2. (2001) International Human Genome Sequencing Consortium. Initial sequencing and analysis of the human genome Nature 409(6822): 860921.

3. Belmont JW, Boudreau A, Leal SM, Hardenbol P, Pasternak S, et al. (2005) A haplotype map of the human genome. Nature 437(7063): 1299-1320.

4. Auton A, Abecasis GR, Altshuler DM, Durbin, RM, Bentley DR, et al. (2015) A global reference for human genetic variation. Nature 526(7571): 6874.

5. Mini E, Nobili S (2009) Pharmacogenetics : implementing personalized medicine. Clinical Cases in Mineral and Bone Metabolism 6(1): 17-24. 
6. Daly AK (2013) Pharmacogenomics of adverse drug reactions. Genome Medicine 5(1): 5 .

7. Lee MTM, Klein TE (2013) Pharmacogenetics of warfarin: challenges and opportunities. Journal of Human Genetics 58(6): 334-338.

8. Caudle KE, Klein TE, Hoffman JM, Müller DJ, Whirl carrillo M, et al. (2014) Incorporation of Pharmacogenomics into Routine Clinical Practice: the Clinical Pharmacogenetics Implementation Consortium (CPIC) Guideline Development Process. Current Drug Metabolism 15(2): 209-217.

9. Mohs RC, Greig NH (2017) Drug discovery and development: Role of basic biological research. Alzheimer's \& Dementia: Translational Research \& Clinical Intervention 3(4): 651-657.

10. Differding E (2017) The Drug Discovery and Development Industry in India- Two Decades of Proprietary Small-Molecule R \& D. 12(11): 786818.

11. Mohs RC, Greig NH (2017) Drug discovery and development: Role of basic biological research. Alzheimer's \& Dementia: Translational Research \& Clinical Interventions 3(4): 651-657.

12. Lihite RJ, Lahkar M, Das S, Hazarika D (2017) A study on adverse drug reactions in a tertiary care hospital of Northeast India. Alexandria Journal of Medicine 53(2): 151-156.

\section{ISSN: 2574-1241}

DOI: $10.26717 / B J S T R .2018 .11 .002073$

Husayn Ahmed P. Biomed J Sci \& Tech Res

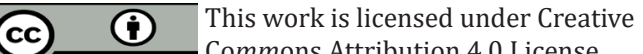

Submission Link: https://biomedres.us/submit-manuscript.php
13. Priyadarshini R, Raj GM, Shewade DG (2018) Status of Pharmacogenomics Research in India During the Last Five Years. Proceedings of the Indian National Science Academy 84(1): 195-223.

14. Li H (2014) Toward better understanding of artifacts in variant calling from high-coverage samples. Bioinformatics 30(20): 2843-2851.

15. Schwarz UI, Gulilat M, Kim RB (2018) The Role of Next-Generation Sequencing in Pharmacogenetics and Pharmacogenomics. Cold Spring Harbor Perspectives in Medicine p. 1-16.

16. Limaye N (2013) Pharmacogenomics, Theranostics and Personalized Medicine - the complexities of clinical trials: Challenges in the developing world. Applied \& Translational Genomics 2: 17-21.

17. Xing J, Watkins WS, Hu Y, Huff CD, Sabo A, et al. (2010) Genetic diversity in India and the inference of Eurasian population expansion. Genome Biology 11(11).

18. Peterson JF, Field JR, Shi Y, Schildcrout JS, Denny JC, et al. (2015) Attitudes of clinicians following large-scale pharmacogenomics implementation. The Pharmacogenomics Journal p. 1-6.

$\begin{array}{ll}\text { BIOMEDICAL } & \text { Assets of Publishing with us } \\ \text { RESEARCHES } & \text { - Global archiving of articles } \\ \text { - Immediate, unrestricted online access }\end{array}$

